\title{
Desenvolvimento de um experimento controlado remotamente e um simulador tridimensional para demonstrar a lei do inverso do quadrado
}

Development of a remotely controlled experiment and a three-dimensional simulator to demonstrate the inverse-square law

\author{
Izac Martins da Silva ${ }^{*} @$, Marisa Almeida Cavalcante ${ }^{2 @}$, Vitor Bremgartner da Frota1@ \\ ${ }^{1}$ Instituto Federal do Amazonas, Manaus, AM, Brasil. \\ ${ }^{2}$ Universidade Federal do Amazonas, Departamento de Física, Manaus, AM, Brasil.
}

\begin{abstract}
Recebido em 12 de novembro de 2021. Revisado em 14 de janeiro de 2022. Aceito em 01 de fevereiro de 2022.
\end{abstract}
\begin{abstract}
Este trabalho apresenta resultados relacionados ao desenvolvimento e validação de um experimento controlado remotamente e um simulador tridimensional, voltados ao estudo prático da lei do inverso do quadrado da distância. As etapas de criação do protótipo consistiram na manufatura dos mecanismos físicos, do hardware e software necessários pela comunicação e controle entre o usuário e o experimento através de um dispositivo com acesso à internet, possibilitando mensurar a intensidade luminosa de uma fonte de luz que se afasta gradativamente de um fotoresistor (LDR, do inglês "Light Dependent Resistor"). Já as etapas de desenvolvimento do simulador foram divididas em modelagem gráfica 3D de todos os componentes e programação da interação, animação e simulação de dados, assim criando o aplicativo para dispositivos Android. A validação do protótipo foi realizada usando análise estatística e a propagação do erro, além de confrontar os dados obtidos com um luxímetro. Os resultados foram satisfatórios para validação do protótipo e da qualidade das ferramentas de prática experimental, em virtude do relativo baixo custo despendido e do seu potencial de uso.
\end{abstract}

Palavras-chave: Experimentação Remota, Simulações tridimensionais, Lei do inverso do quadrado.

This work presents results related to the development and validation of a remotely controlled experiment and a three-dimensional simulator, aimed at the practical study of the inverse square law of distance. The prototype creation steps consisted in the manufacture of the physical mechanisms, hardware and software necessary for the communication and control between the user and the experiment through a device with internet access, making it possible to measure the luminous intensity of a light source that gradually moves away of a photoresistor (LDR, "Light Dependent Resistor"). The simulator development stages were divided into 3D graphic modeling of all components and interaction programming, animation and data simulation, thus creating the application for Android devices. The prototype validation was performed using statistical analysis and error propagation, in addition to comparing the data obtained with a lux meter. The results were satisfactory for the validation of the prototype and the quality of the experimental practice tools, due to the relative low cost spent and its potential for use.

Keywords: Remote Experimentation, Three-dimensional simulations, Inverse square law.

\section{Introdução}

O Ensino no mundo, assim como no Brasil, está passando por muitas mudanças em função da pandemia de COVID-19, que forçou o distanciamento social e modificou a realidade de milhões de alunos e professores, alertando sobre a importância de sistemas e ferramentas que possibilitem a continuação das atividades acadêmicas através do ensino remoto [1, análogo a modalidade de Educação a Distância (EAD), que utiliza Ambiente Virtual de Aprendizagem (AVA) como interface entre os professores e alunos [2].

\footnotetext{
*Endereço de correspondência: izacmsilva@gmail.com
}

Contudo, mesmo com o término dessa época de distanciamento social, é possível que muitas instituições de ensino no país tendem a incorporar parcialmente procedimentos e práticas aplicadas durante esse período, convergindo potencialmente para um ensino híbrido [3], que através de uma metodologia intencional e ativa bem definida, mescle atividades presenciais e online, centrando o processo de aprendizagem no aluno e mediado pelo professor [4 6 .

É interessante observar algumas formas distintas de implementação de práticas experimentais, voltadas ao ensino de Física, caracterizando-as em função do tipo de acesso: Laboratórios Tradicionais (LT), com a utilização ou criação de experimentos montados e/ou operados presencialmente pelos alunos [7] Laboratórios Remotos 
(LR), os quais permitem interação e visualização de experimentos reais, mas controlados remotamente [8]10]; Simuladores Virtuais (SV), que demonstram uma aproximação dos experimentos reais, com potencial de visualização e interação superior às anteriores, mas com ressalvas aos seus dados, por se tratar de um modelo da realidade [11-13. Para a melhoria do processo de ensino-aprendizagem, a prática experimental como um todo, tem seus resultados positivos, independente se são LT, LR ou SV [7, 14].

Portanto, é importante fomentar o desenvolvimento e implementação, de novas ferramentas de prática experimental, que possam ser usadas em metodologias ativas, adaptáveis ao ensino presencial ou à distância, podendo ser utilizado por dispositivos de Tecnologia da Informação e Comunicação (TICs), mais populares entre os alunos [15], permitindo a visualização de experimentos que ajudem na compreensão de fenômenos físicos e a obtenção de dados confiáveis, independentes de espaço e tempo.

Logo, o objetivo desse trabalho é apresentar o desenvolvimento de um experimento que pode ser usado como LT, mas é adaptado para um LR e replicado em SV, com o tema de estudo da Lei do Inverso do Quadrado da Distância (LIQD) [16, 17] e usando como base trabalhos que estudam formas de detecção da variação da intensidade luminosa [18 21], com diretrizes de maximização de qualidade pelo menor custo, disponibilização gratuita e automatizada para o uso ininterrupto dos artefatos produzidos. As ferramentas criadas são recomendadas para o ensino híbrido, por possuírem atributos que permitem a sua implementação em todos os Modelos de Rotação da Zona Híbrida [3], mas também podendo ser aplicadas em modelos presenciais ou remotos.

A estrutura do presente artigo é composta pela seção 2 tratando da base teórica que o experimento aborda; nas seções 3 e 4 serão apresentadas a concepção e criação dos protótipos de LR e SV; na seção 5. o protótipo passa pelo processo de validação através da análise estatística dos erros; na seção 6, apresentamos as considerações finais.

\section{Fundamentação Teórica}

Existe uma importante relação matemática que se repete em vários ramos de estudo da Física, dando sustentação para diferentes temas, com abordagem tanto no ensino básico como no superior, como por exemplo, a Gravitação Universal, em que o módulo da força de atração entre duas massas, é proporcional ao produto entre elas e inversamente proporcional ao quadrado da distância. Da mesma forma, a Lei de Coulomb é definida como o módulo da força elétrica resultante entre duas cargas, sendo proporcional ao produto delas, e inversamente proporcional ao quadrado da distância entre elas.

Outra área da Física que utiliza essa relação é o estudo da intensidade da radiação de partes do espectro
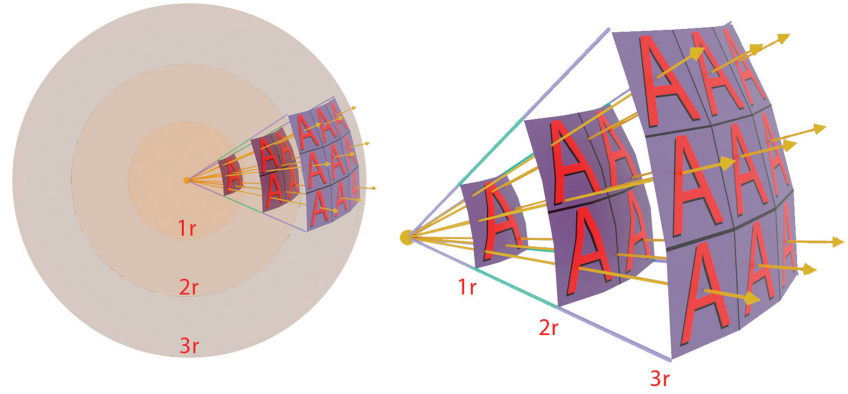

Figura 1: À esquerda, uma fonte de radiação delimitada por três esferas concêntricas e com raio linearmente crescente; à direita, uma seção de um ângulo sólido da fonte de radiação e as três frações das esferas delimitadoras.

eletromagnético, que relaciona a energia emitida por unidade de tempo e o quadrado da distância, seja na seção não-ionizante, quanto para ionizante. Um exemplo da aplicação do primeiro caso é dado pela relação entre as diferentes intensidades da luz visível do sol em diferentes planetas do sistema solar. Em relação à radiação ionizante, pode-se exemplificar através da aplicação na física médica, como parte integrante do estudo da camada semi-redutora de radiação para proteção e segurança no uso de equipamentos médicos, baseados na emissão de radiação ionizante [22].

Se analisarmos as leis anteriores considerando que os coeficientes de proporcionalidade e as demais variáveis, excluindo a distância, permanecem constantes, nos resta uma relação matemática. A ela denominamos como a Lei do Inverso do Quadrado da Distância (LIQD) [16, 17, equação (1).

$$
I \propto d^{-2}
$$

Observando pelo prisma de uma fonte emissora de radiação, $I$ é a intensidade dessa radiação e d é sua distância, logo, a radiação emitida, vai se espalhando uniformemente no espaço em função do afastamento entre a fonte e o ponto de observação, com isso seu módulo decai como mostrado na Figura 1

Como o fluxo de radiação luminosa que ultrapassa as áreas das três seções é constante, sua densidade diminui em função do aumento da área seguindo a LIQD. Esse fenômeno pretende ser demonstrado experimentalmente através do protótipo proposto, em que uma fonte de luz se afastará gradativamente de um fotossensor e com o registro de cada distância feita ao longo do processo, a área de superfície do sensor permanece constante, sendo assim esperado um decaimento exponencial da intensidade com o aumento da distância, análogo à Figura 1 lado direito.

\section{Experimentação Remota}

A principal referência à construção desse protótipo foi o RExLab [23, que há mais de duas décadas desenvolve 
trabalhos na área de experimentação remota no Brasil. Assim, nossas diretrizes foram norteadas pela relação da melhor qualidade em função do menor custo, ocasionando várias versões diferentes dos equipamentos e tecnologias envolvidas no seu desenvolvimento.

Sua versão estável é dividida em três partes, inicialmente a estrutura física do protótipo e seus mecanismos, em seguida o hardware e o funcionamento esperado do experimento e por fim a automação e controle remoto na aplicação web.

\subsection{Estrutura do protótipo}

A construção do artefato físico foi realizada com o uso de materiais de baixo valor econômico e recicláveis, que possam ser reaproveitados no futuro, dando uma característica potencialmente mutável para o experimento. Foram usadas garrafas pets, tubos e conexões de PVC soldáveis de 100, 25 e $20 \mathrm{~mm}$ (Figura 2), entretanto, o encaixe foi feito somente por interferência, sem o uso de resinas, para possibilitar a reestruturação do esqueleto do experimento, se necessário.

A estrutura do experimento consiste em duas partes, uma fixa, que acomoda o hardware, e dá sustentação para todo experimento, servindo também de guia para a segunda, a parte móvel, responsável pela elevação vertical da fonte luminosa. O resultado da manufatura estrutural pode ser visto na Figura 3.

O mecanismo de elevação, apresentado na Figura 4 , funciona quando o usuário aciona o motor, rotacionando o carretel (iv) e enrolando o fio, representado pela cor azul da figura, passando pela roldana inferior (ii), até a conexão no sistema de contrapeso (iii) e no fio elétrico resistente à tração, representado pela cor preta. Esse cabo passa pelas duas roldanas superiores (ii), ligandoo ao sistema móvel da fonte luminosa (i). A massa do sistema móvel luminoso é um pouco superior do que a do contrapeso, logo, possibilitando o retorno à posição inicial, por gravidade com a suave reversão do motor.

Em função dos sensores, o dimensionamento de forças estáticas e dinâmicas, assim como possíveis elasticidades dos fios, não se faz necessário para futura obtenção de dados.

\section{Canos de $100 \mathrm{~mm}$}

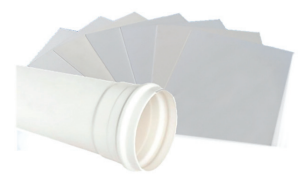

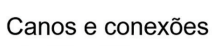
de 20 e $25 \mathrm{~mm}$

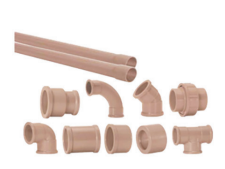

Garrafas Pet

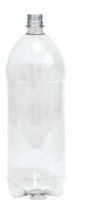

Figura 2: Materiais usados na construção da estrutura do experimento.

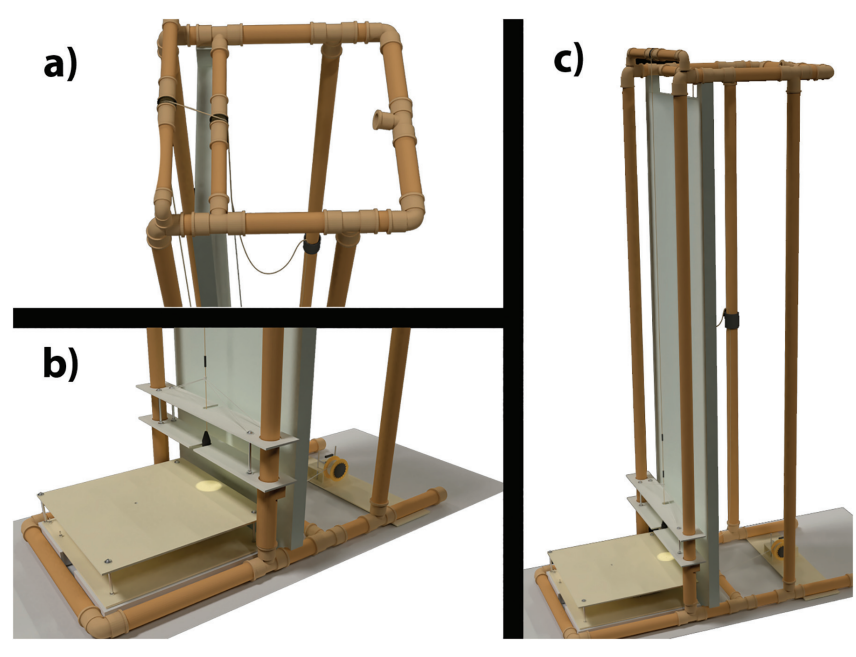

Figura 3: a) vista superior angular esquerda. b) vista lateral inferior esquerda. c) Visão panorâmica do experimento.

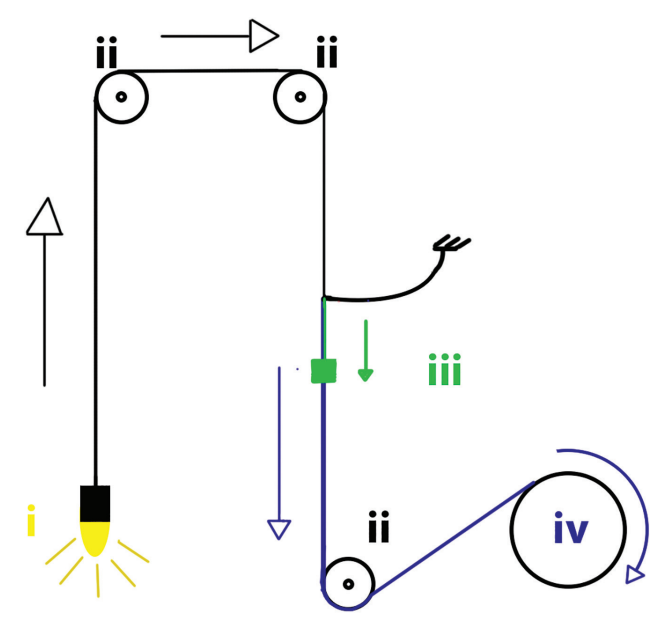

Figura 4: i) sistema móvel da fonte luminosa; ii) roldanas feitas de garrafas pet; iii) contrapeso do sistema de elevação; iv) carretel de enrolamento do fio.

\subsection{O hardware utilizado}

Os demais itens utilizados são componentes eletrônicos, dentre eles usamos uma placa de Arduino; uma protoboard; um LDR; uma lâmpada LED; um LED vermelho; alguns resistores; alguns jumpers; um controlador de ponte H L298n; um sensor de distância ultrassônico HC-SR04; um motor de carro de controle remoto e um Raspberry Pi 3, como podemos ver na Figura 5.

Ao contrário da grande parte dos trabalhos que utilizam Arduino como ferramenta de coleta de dados [14, 18, 20, 21, 24], inclusive os que o fazem remotamente [8, 9. 14, 25], nesse projeto ele tem sua responsabilidade limitada pela comunicação indireta com os sensores e atuadores, mas toda a responsabilidade de controle é delegada ao servidor web, no Raspberry $\mathrm{Pi} 3$, que será detalhado posteriormente. Contudo, os esquemas de conexões elétricas entre o Arduino e seus periféricos são descritos na Figura 6 . 


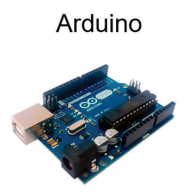

Webcam

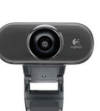

Ponte H I298n

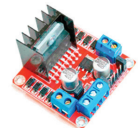

Protoboarde
componentes

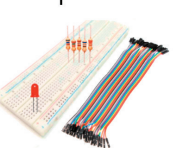

Ultrassônico

$\mathrm{HC}-\mathrm{SRO} 4$

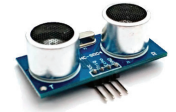

Lâmpada e LDR

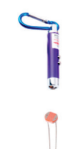

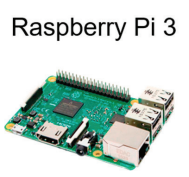

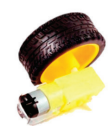

Roda com motor

Figura 5: Componentes eletrônicos usados no hardware do experimento.

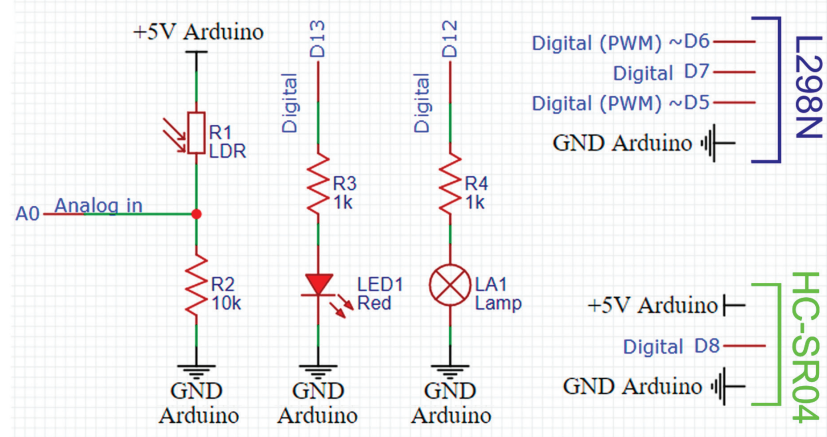

Figura 6: Esquema de ligação dos pinos do Arduino com os sensores e atuadores do circuito.

A primeira parte desse circuito é o divisor de tensão entre o fotoresistor LDR e um resistor de $10 k \Omega$, mensurado através da porta $\mathrm{A} 0$, seguido pelo simples sistema luminoso de um LED (porta D13), que alerta quando a intensidade luminosa é inferior a um parâmetro determinado pelo usuário. Já o controle da fonte luminosa, também pelo usuário, é feito através da porta D12.

O sistema dinâmico do projeto usa um módulo de ponte H, o drive L298n, que é responsável pela rotação em ambos os sentidos e variação de potência do motor, o qual controla a parte móvel, permitindo a subida e descida da fonte luminosa. Contudo, é necessária sua própria fonte de alimentação externa de $12 \mathrm{~V}$, para não sobrecarregar a tensão do sistema, o que poderia gerar uma queda de tensão da seção responsável pela leitura de dados oriundos do LDR, logo, comprometendo a integridade dos dados gerados pelo experimento. Sua ligação é esquematizada na Figura 7.

Para medir o intervalo de distância entre a fonte luminosa e o LDR é usado o sensor ultrassônico HC-SR04, que permite uma tolerância maior para calibração inicial do experimento e reduz a necessidade de manutenção externas. Sua montagem é esquematizada na Figura 8

\subsection{Aplicação Web e automação}

É entendido como aplicação Web um sistema projetado para utilização através de um navegador de Internet,

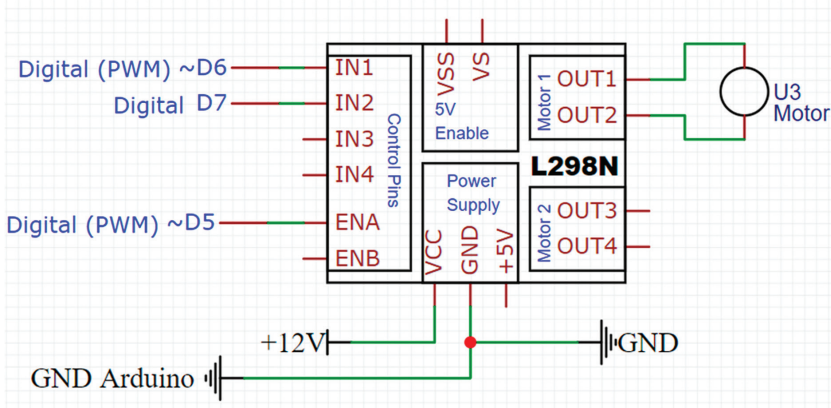

Figura 7: Esquema de montagem da ponte H L298N com o Arduino Uno.

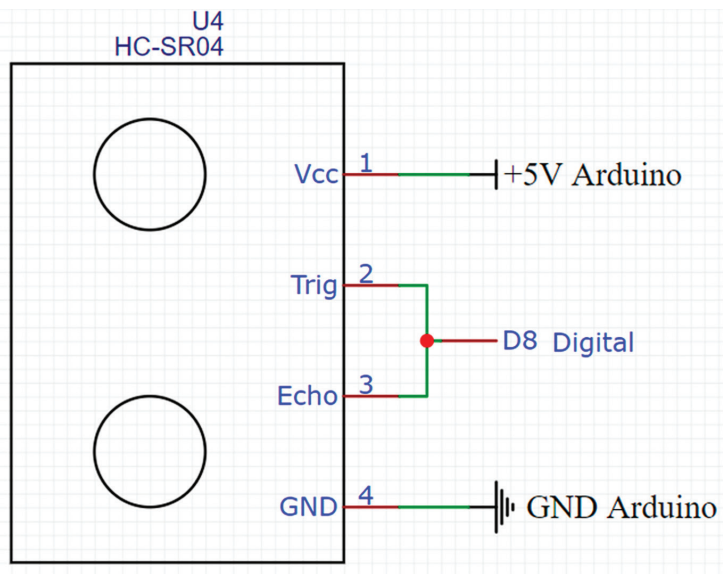

Figura 8: Conexões entre o sensor de distância ultrassônico HCSR04 e o Arduino.

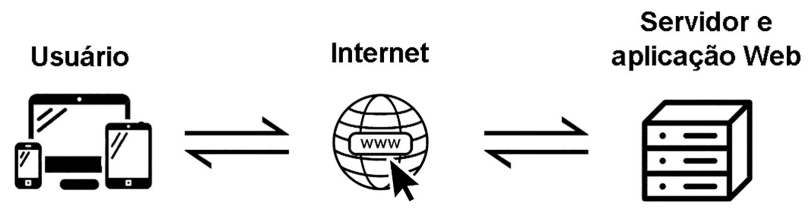

Figura 9: Configuração básica de um servidor e uma aplicação web.

podendo ser acessado por qualquer dispositivo conectado à rede como computadores, tablets, smartphones, smarTVs etc.

Foram feitas diferentes versões usando vários softwares, hardwares e sistemas operacionais, resultando na configuração estável exposta no sítio [26] (Figura 9], que lista todas as tecnologias envolvidas para um olhar mais profundo.

Em síntese o servidor foi desenvolvido em Node.JS, usando a linguagem de programação JavaScript (JS), com a Linguagem de Marcação de HiperTexto HTML, responsável pela estrutura da página e Cascading Style Sheet (CSS), para adicionar estilos no documento web. Além de toda estrutura do servidor, o sistema controla os sensores e atuadores do Arduino, com um código já existente na sua IDE (Ambiente de Desenvolvimento 


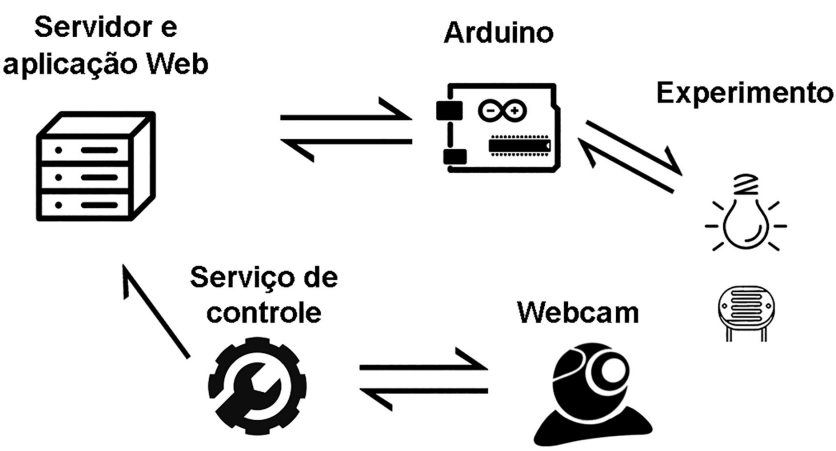

Figura 10: Controle do servidor web sobre os periféricos.

Integrado), que possibilita essa conexão entre um servidor e a placa de prototipagem de forma simples, através da ativação do código Standard Firmata [27].

Os Códigos de desenvolvimento do experimento controlado remotamente JS, HTML e CSS 28] consistem em poucas linhas para criar o servidor, conectar com o Arduino e controlar o experimento, recebendo de volta informações que são tratadas, organizadas e exibidas em tempo real para o usuário (Figura 9 e Figura 10). Com o término do procedimento experimental, o aluno pode exportar os dados e trabalhar como for recomendado pelo professor, podendo utilizá-los diretamente no software Excel para plotar diversos gráficos dependendo da finalidade escolhida no plano de ensino-aprendizagem da aula.

O sistema opera autonomamente e garante reinicialização em casos de queda de energia, ou em erros críticos no servidor, voltando a manter conexão com a rede sem intervenção técnica.

O valor do protótipo foi de aproximadamente U\$ 200, entre componentes mecânicos e eletrônicos, tendo um custo relativamente baixo em função do potencial ofertado da ferramenta aos professores e alunos.

\subsection{Funcionamento do experimento real}

Unindo todas as etapas anteriores o protótipo é gerado e funciona da seguinte forma: quando acionado o Raspberry se comunicará com o Arduino, que por sua vez acionará a fonte luminosa, o LDR retornará o valor da intensidade da medida analógica oriunda de um divisor de tensão entre o LDR e um resistor simples, paralelamente o sensor ultrassônico retorna a distância entre a fonte e o sensor de luz. Em seguida o motor é acionado fazendo com que a estrutura móvel, na qual a fonte luminosa está fixada, eleve-se pausadamente, aumentando a distância entre a lâmpada e o resistor dependente de luz linearmente. Esse processo repetese a cada 2 segundos, por 24 iterações. Assim, com os dados primários, são calculadas todas as variáveis que forem convenientes, sendo enviados ao solicitante em tempo real. Ao término do experimento o sistema volta à posição inicial e se autocalibra para próxima utilização.
A Figura 6, lado esquerdo, mostra o circuito que recebe um sinal analógico periódico, oriunda do divisor de tensão (porta A0). Paralelamente também temos um sistema de alerta luminoso (porta D13), em percentual de luminosidade, para constatação visual de parâmetros iniciais pelo usuário, enquanto a fonte luminosa (porta D12) se distancia do sensor periodicamente, o valor analógico flutua entre 0 e 1023, que é relacionável diretamente entre 0 e $5 \mathrm{~V}$. Logo, todo o cálculo das variáveis é realizado em função do módulo lido do LDR, e relativo a uma distância específica, obtida pelo sensor HC-SR04.

Em função da ordem escolhida entre o LDR e o resistor $R_{2}$, é realizado uma regra de três simples, entre os valores máximos e mínimos para equacionar a tensão em $R_{2}$, assim pode-se chegar na tensão $U_{L D R}$ em Volts, como vista na equação (2).

$$
U_{L D R}=5\left(1-\frac{N}{1023}\right)
$$

O $N$ é o valor analógico $(0 \sim 1023)$ lido pelo na porta $A 0$. Como a corrente é a mesma em todos os pontos envolvidos nesse circuito e sabendo os valores $U_{R_{2}}$ e $R_{2}=10 \mathrm{k} \Omega$, é possível calcular facilmente a corrente pela equação 3 .

$$
i=i_{R_{2}}=i_{L D R}=\frac{N}{2046}
$$

De posse de todas as tensões, e as correntes envolvidas, é possível relacionar as equações e chegar na resistência relativa no LDR $R_{L D R}$, através da equação (4).

$$
R_{L D R}=10\left(\frac{1023}{N}-1\right)
$$

Em seguida, é feita uma análise gráfica em escala logarítmica, baseado em Santos [29], considerado linear a relação da resistência em função da intensidade luminosa no intervalo fechado de 10 a $100 l x$, como visto na Figura 11

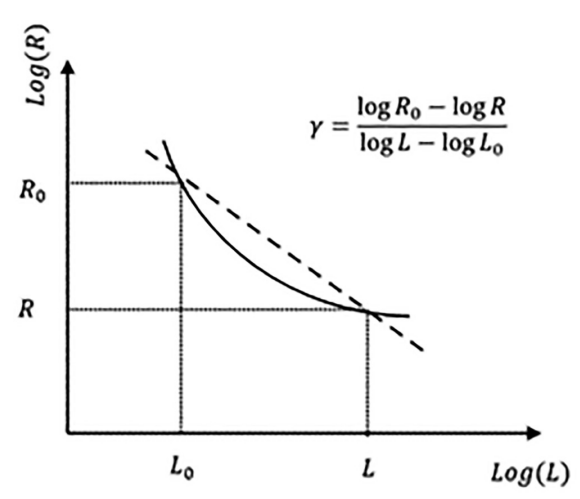

Figura 11: Relação gráfica entre a resistência e a intensidade luminosa de um LDR na escala logarítmica. 

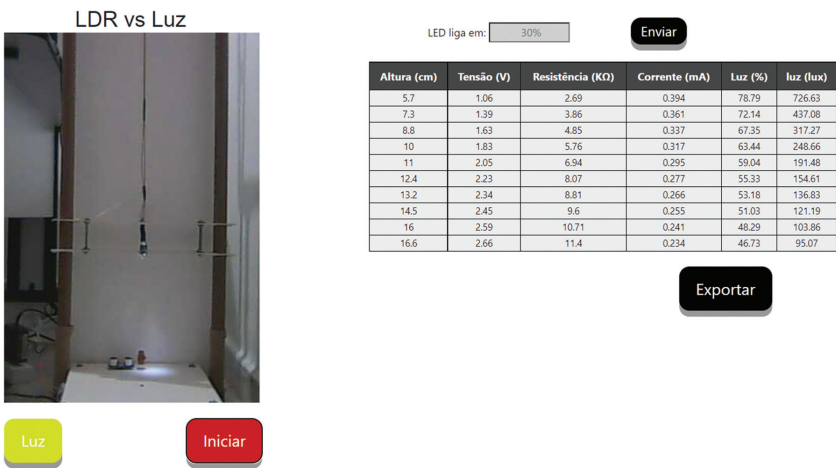

Figura 12: Interface do experimento remoto para um computador.

Logo, chegando na equação (5), que é uma aproximação para $L_{L D R}$ em lux 17 em que $L_{0}=10 l x$ é a intensidade luminosa inicial, $R_{0}=56 k \Omega$ é a resistência equivalente e $\gamma=0,71$ é a taxa de variação. Todos os valores podem ser obtidos no datasheet do fabricante do LDR, contudo para maior exatidão, o componente foi submetido a testes, para mensurar as resistências relativas aos dois valores extremos de intensidade luminosa, assim calculando seu $\gamma_{100 / 10}$, pelo $\log \left(R_{L_{100}} / R_{L_{10}}\right)$. Os dados foram coletados do LDR usando um Luxímetro Hikari HLX-912 e um multímetro Hikari HM-1001, com 24 ciclos de repetição, a uma temperatura de $30,1^{\circ} \mathrm{C}$.

$$
\begin{gathered}
L=L_{0} \cdot R_{0}^{\left(\frac{1}{\gamma}\right)} \cdot R^{\left(-\frac{1}{\gamma}\right)} \\
L=2933,4788\left(\left(\frac{1023}{N}-1\right) 10\right)^{(-1,40906)}
\end{gathered}
$$

Por fim, podendo comparar as distâncias medidas com as suas respectivas intensidades luminosas, para encontrar a função que mais se aproxima, comparandoas com a LIQD, chegamos na equação (6).

$$
L=\alpha \cdot d^{n}
$$

O $\alpha$ é um coeficiente de proporcionalidade e $n$ é o expoente da variável, ambos retirados da regressão dos dados obtidos experimentalmente, logo, a confirmação da Lei do Inverso do Quadrado da Distância pode ser confrontada.

Na Figura 12 é apresentada a interface de utilização para computador ao longo do experimento, assim como os dados sendo medidos, coletados e disponibilizados em tempo real.

\section{Simulador Virtual}

O processo de concepção do simulador que reproduz fielmente o experimento anteriormente mostrado, será

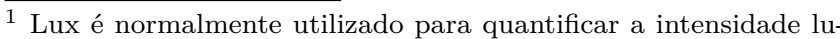
minosa observável em uma superfície, ou seja, pode ser classificado como a densidade do fluxo luminoso, relativo a uma área incidente, sua unidade de medida é $\mathrm{lx}$.
}

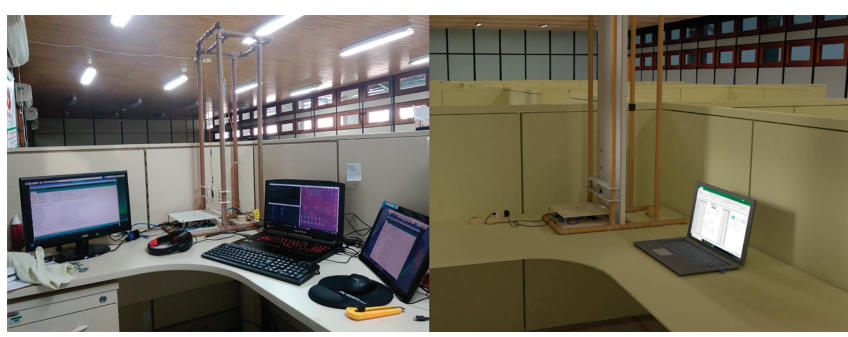

Figura 13: Imagem do protótipo real e renderização do modelo virtual, respectivamente.

descrito da mesma forma que a criação da aplicação web, expondo as principais tecnologias aplicadas de forma sucinta. O formato escolhido do protótipo é de aplicativo Android, pois é o sistema operacional mais popular entre os dispositivos móveis no Brasil, tendo compatibilidade com qualquer sistema igual ou superior ao Android 4.1.

\subsection{Modelagem gráfica}

O Software Blender 30 foi escolhido como principal ferramenta de modelagem por ser open-source com todos os componentes necessários de distribuição gratuita, possibilitando a produção de modelos 3D, animações, simulações físicas, edição de vídeo, texturização, renderização e jogos, tudo isso com alta qualidade.

Esse processo, em síntese, consiste na criação de todos os componentes do experimento real de forma virtual, passando pela modelagem geométrica, texturização de materiais, animações de movimento, renderização de imagens, criação de vídeos e exportação de modelos. $\mathrm{O}$ resultado alcançado pode ser observado na Figura 13. com um ótimo nível de renderização em função da gratuidade da ferramenta, demandando somete tempo de trabalho para criação de material gráfico 3D com seu uso.

\subsection{Criação de simuladores $3 \mathrm{D}$}

A principal ferramenta usada nessa etapa é o Unity 3D [31, que importa do Blender, os modelos anteriormente gerados. Ela é uma importante aliada na criação de jogos e simuladores para as mais diversas plataformas do mercado, como Windows, Linux, Mac, Android, iOS, Xbox, Playstation, entre outros. Dando a possibilidade de programar em duas linguagens, C\# ou Javascript, o software possui uma extensa gama de documentação gratuita na web. Suas etapas de desenvolvimento didático obedecem às fases de criação de um jogo educacional ou simulador [32, 33]: análise do tema; delimitação do projeto, desenvolvimento técnico, testes preliminares, avaliação e publicação.

Em síntese, foi usada a linguagem $\mathrm{C}$ \# para criação de toda a interação, apresentação do conteúdo e animação usados no simulador. Os dados são obtidos através de uma prévia coleta do experimento real e atribuindo um 


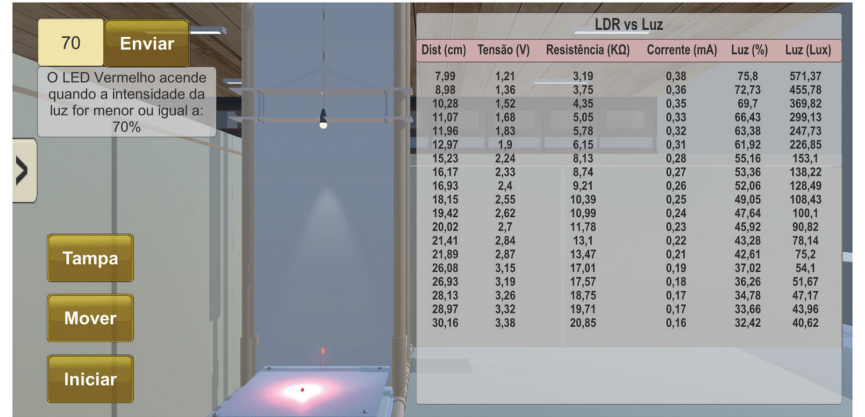

Figura 14: Interface do APP.

erro randômico de $\pm 0,02 \%$ para cada medida fundamental, logo as equações são igualmente usadas nesse processo (Figura 14).

Depois da criação do arquivo de instalação do aplicativo (APK), passamos para a fase de publicação na Play Store que gerou o único custo relevante do projeto virtual (US\$ 50). Dessa forma, foi possível disponibilizar o aplicativo para download [34], que já teve centenas de usuários em vários países, como Brasil, Índia, Singapura, China, Ucrânia, França, Marrocos, Costa do Marfim, entre outros.

O experimento virtual possui algumas vantagens como a possibilidade de execução concomitantemente por vários alunos, com ou sem acesso à Internet, desde que tenha o aplicativo instalado previamente. Outra disponibilidade que a parte virtual permite é a visualização de todos os componentes usados no trabalho, conectados da mesma forma que no real. A listagem e descrição de todas as tecnologias usadas nas fases de modelagem e criação do simulador, estão disponíveis no sítio [35].

\section{Validação do Protótipo}

Para avaliar o artefato real produzido 8, usamos a análise estatística, sendo possível determinar os módulos das médias, suas incertezas associadas e propagação do erro, assim podendo comprovar ou não sua eficiência para atividades experimentais.

Dois dados são coletados pelos sensores: distância e intensidade analógica do divisor de tensão, em cada uma das 24 iterações do experimento. Esse processo foi repetido 30 vezes, gerando uma média para cada um dos dados, usando a equação (7).

$$
\bar{y}=\frac{1}{n} \sum_{i=1}^{n} y_{i}
$$

Em seguida, o valor do desvio padrão do valor médio é obtido, através da equação (8):

$$
\sigma_{m}=\sqrt{\frac{1}{n(n-1)} \sum_{i=1}^{n}\left(y_{i}-\bar{y}\right)^{2}}
$$

Nesse ponto é usado o valor tabelado dos intervalos de confiança para um número de medições pequenas, com nível de confiança de $95 \%$, logo, sendo atribuído um fator de multiplicação para o desvio padrão experimental, relativo ao intervalo de confiança tabelado de $2,13 \sigma_{m}$.

A incerteza residual definida pelos fabricantes é $\sigma_{r_{d}}=$ $\pm 0,3 \mathrm{~cm}$ para o sensor de distância e $\sigma_{r_{A 0}}= \pm 1$ para a porta analógica de 10 bits. Portanto, é possível determinar a incerteza padrão final pela equação (9).

$$
\sigma_{f}=\sqrt{\left(\sigma_{m}\right)^{2}+\left(\sigma_{r}\right)^{2}}
$$

Contudo, como as variáveis medidas são usadas para efetuar diversos cálculos, foi aplicada a propagação do erro para determinar $\sigma_{f}$ para todas as variáveis dependentes através da equação 10 .

$$
\sigma_{f}=\sqrt{\left(\frac{\partial f}{\partial x}\right)^{2} \cdot \sigma_{x}^{2}+\left(\frac{\partial f}{\partial y}\right)^{2} \cdot \sigma_{y}^{2} \cdot \ldots}
$$

Dentre elas destaca-se o resultado da intensidade luminosa calculada pelo sistema $L_{L D R}=\left(\bar{L} \pm \sigma_{L}\right) l x$ equação 11 .

$$
\begin{aligned}
L_{L D R}= & \left(2933,4788\left(\frac{10230}{\bar{N}}-10\right)^{-1,41}\right. \\
& \pm \frac{230,24}{10^{3}}\left(\frac{10230}{\bar{N}}-10\right)^{-1,41}\left(\frac{996898,41}{\bar{N}^{2}}\right. \\
& \left.\left.\cdot\left(\left(\frac{1023}{\bar{N}}-1\right)^{-2}+1\right)+2500\right)^{\frac{1}{2}}\right) l x
\end{aligned}
$$

Por outro lado, a distância respectiva é independente, pois é obtida diretamente. Seu módulo corresponde a $d=(\bar{d} \pm 0,3) \mathrm{cm}$. Os resultados obtidos podem ser observados na Tabela 1] que possui as médias e erros associados das 24 iterações feitas para cada uma das 30 distâncias distintas.

A equação que satisfaz os pontos amostrais surge da plotagem e análise dos dados. Logo, obtemos a equação 12 .

$$
L_{L D R}=30239 d^{-1,925}
$$

O valor 30239 é o coeficiente de proporcionalidade, e o expoente da distância se aproxima do esperado pela LIQD. A forte correlação de $R^{2}=0,9986$, gerada entre os pontos experimentais e a linha de tendencia do decaimento aproximadamente quadrático, evidenciado no gráfico logarítmico apresentado na Figura 15

Por outro lado, com o intuito de confrontar os resultados, foi usado o luxímetro Hikari HLX-912, que possui uma incerteza associada de $4 \%$ para cada medida menor que $10.000 \mathrm{~lx}$, onde foram obtidas 10 iterações relativas para cada uma das 25 distâncias distintas, chegando na equação 13 :

$$
L_{H L X}=32277 d^{-1,994}
$$


Tabela 1: Tabela com os resultados das médias experimentais da distância e seu erro associado e as respectivas intensidades luminosas e seu erro propagado.

\begin{tabular}{lcccc|ccccc}
\hline$n^{\mathrm{O}}$ & $\bar{d}(\mathrm{~cm})$ & $\sigma_{d}(\mathrm{~cm})$ & $\bar{L}_{L D R}(l x)$ & $\sigma_{L_{L D R}}(l x)$ & $n^{\mathrm{O}}$ & $\bar{d}(\mathrm{~cm})$ & $\sigma_{d}(\mathrm{~cm})$ & $\bar{L}_{L D R}(l x)$ & $\sigma_{L_{L D R}}(l x)$ \\
\hline 1 & 8,1 & 0,3 & 570,71 & 40,35 & 16 & 27,1 & 0,3 & 51,62 & 3,64 \\
2 & 9,1 & 0,3 & 455,29 & 32,17 & 17 & 28,2 & 0,3 & 47,12 & 3,33 \\
3 & 10,3 & 0,3 & 369,44 & 26,09 & 18 & 29,0 & 0,3 & 43,92 & 3,10 \\
4 & 11,1 & 0,3 & 298,84 & 21,10 & 19 & 30,2 & 0,3 & 40,58 & 2,87 \\
5 & 12,0 & 0,3 & 247,50 & 17,47 & 20 & 31,1 & 0,3 & 39,93 & 2,82 \\
6 & 13,0 & 0,3 & 226,64 & 16,00 & 21 & 32,0 & 0,3 & 37,61 & 2,66 \\
7 & 15,3 & 0,3 & 152,96 & 10,79 & 22 & 33,5 & 0,3 & 35,96 & 2,54 \\
8 & 16,2 & 0,3 & 138,10 & 9,74 & 23 & 34,2 & 0,3 & 33,56 & 2,37 \\
9 & 17,0 & 0,3 & 128,38 & 9,06 & 24 & 35,4 & 0,3 & 30,97 & 2,19 \\
10 & 18,2 & 0,3 & 108,34 & 7,64 & 25 & 36,3 & 0,3 & 31,10 & 2,20 \\
11 & 19,5 & 0,3 & 100,01 & 7,06 & 26 & 37,3 & 0,3 & 29,97 & 2,12 \\
12 & 20,1 & 0,3 & 90,74 & 6,40 & 27 & 38,2 & 0,3 & 28,26 & 2,00 \\
13 & 21,5 & 0,3 & 78,08 & 5,51 & 28 & 39,1 & 0,3 & 27,29 & 1,93 \\
14 & 22,0 & 0,3 & 75,13 & 5,30 & 29 & 40,4 & 0,3 & 25,86 & 1,83 \\
15 & 26,1 & 0,3 & 54,05 & 3,82 & 30 & 41,0 & 0,3 & 25,30 & 1,79 \\
\hline
\end{tabular}

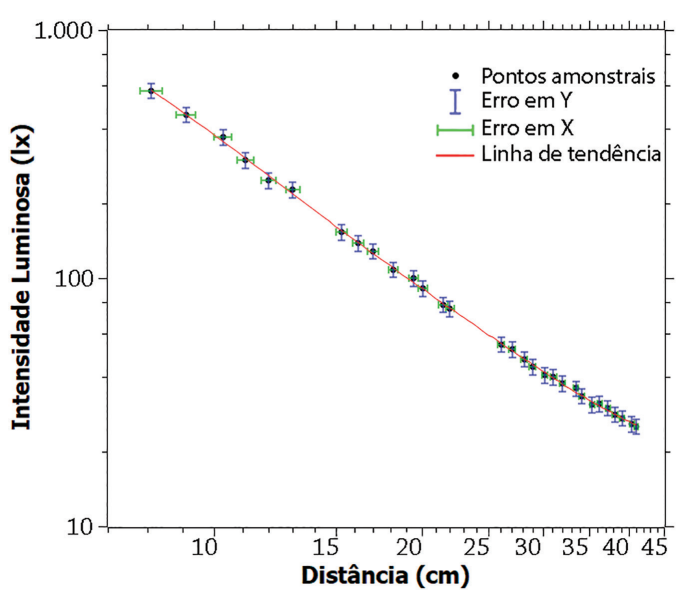

Figura 15: Gráfico em escala logarítmica da Intensidade Luminosa em função da distância.

Comparando os resultados entre o protótipo experimental e um luxímetro, é possível constatar uma aproximação dos valores obtidos pelo protótipo de $96,5 \%$ em relação ao equipamento de medição, assim como um erro associado de aproximadamente de $7 \%$ do valor mensurado, o que não está muito diferente do luxímetro profissional HLX com seus $4 \%$ de erro.

Já o simulador toma como parâmetros os dados obtidos em várias realizações do experimento real, para geração de dados simulados.

\section{Considerações Finais}

Neste artigo demonstramos o processo de desenvolvimento e validação de um experimento do inverso do quadrado da distância, controlado remotamente e um simulador que o reproduz virtualmente. Embora tenham sido idealizadas para uso no modelo híbrido de ensino, não são limitadas. Essas ferramentas podem ser adaptadas para aulas presenciais, remotas, estudos autônomos até para uso offline do simulador em regiões sem conexão com Internet.

Destaca-se sobre simulador duas formas diferentes para sua obtenção, além de estar disponível na Google Play Store, com o título LDR vs Luz; há também a possibilidade de obtenção do arquivo APK, possibilitando a instalação manualmente nos dispositivos móveis que serão usados nas aulas práticas. Portanto, visamos fomentar seu uso em vários contextos de aprendizagem com realidades distintas, proporcionando aos docentes liberdade de espaço e tempo para implementação da ferramenta, podendo ser presencial ou remota, online ou offline, síncrona ou assíncrona.

Em ambientes ideais, com acesso à Internet e disponibilidade de celulares ou computadores, recomendamos a implementação das ferramentas conjuntamente, como uma forma de complementação para otimizar o tempo limitado das aulas práticas sobre o tema. O simulador pode proporcionar nos alunos o primeiro contato com o experimento proposto, facilitando a compreensão do seu funcionamento pela interação e repetição, possibilitando a diminuição do tempo necessário para realização do experimento controlado remotamente. Esse tempo é um dos principais pontos sensíveis na sua implementação, pois há somente uma instância do experimento real disponibilizada, limitando os acessos simultâneos. Logo, para realização da atividade é estipulado de 1 até 3 minutos para coleta de dados, cabendo ao professor planejar sua aula em função da quantidade de alunos e tempo disponível.

O custo de produção total das ferramentas foi de U\$250, contudo quando observado o potencial de uso e a qualidade final entregues à sociedade, pode-se considerar extremamente baixo em função da quantidade de pessoas, de diferentes regiões no mundo, que podem usufruir dessas ferramentas.

Atualmente estamos trabalhando em uma nova versão do experimento remoto [36], tornando-o mais robusta e resistente, assim como a padronização do hardware 
e software com RExLab [23, o que proporcionará a indexação do trabalho com a sua plataforma de referência internacional, sendo disponibilizada ao público assim que possível. Também está em desenvolvimento um produto educacional com recomendações de implementações das ferramentas integradas a metodologia ativa da Aprendizagem Baseado em Projeto (ABP), voltados para o ensino no nível médio, pois o desejo dos autores é incentivar a criação e o uso de ferramentas de prática experimental que gratuitamente ofereçam oportunidade de enriquecer o processo de ensino-aprendizagem independentemente da localização física dos sujeitos envolvidos.

\section{Agradecimentos}

Agradecemos primeiramente ao apoio do $\mathrm{CNPq}, \mathrm{CA}-$ PES e Fundação de Amparo à Pesquisa do Estado do Amazonas (FAPEAM), que proporcionaram, em momentos distintos, o desenvolvimento desse projeto. Também gostaríamos de agradecer os professores que sempre estiveram dispostos a ajudar e contribuir direta e indiretamente com o projeto: Aroldo de Almeida Guerreiro, Yuri Expósito Nicot, Leandro Galvão e Rui Batista. A toda equipe do RExLab da UFSC em especial ao professor Juarez Bento da Silva que deram suporte técnico. Ao Instituto de Ciências Exatas e o Instituto de Computação da UFAM, assim como o Programa de Pósgraduação em Ensino Tecnológico do IFAM (PPGET).

\section{Referências}

[1] B.S. Paula, C. Codeço, M. Hor-Meyll e T. Paiva, Rev. Bras. Ens. Fis. 43, e20200518 (2021).

[2] J. Mattar, L.M.M. Rodrigues, W. Czeszak e J. Graciani, Educação em Revista 36, e217439 (2020).

[3] C.M. Christensen, M.B. Horn e H. Staker, Ensino híbrido: uma inovação disruptiva? Uma introdução à teoria dos híbridos (Clayton Christensen Institute, Boston, 2013), v. 1, p. 52.

[4] F.F. Monteiro, Rev. Bras. Ens. Fis. 43, e20200315 (2021).

[5] M.A. Cavalcante e E. Molisani, Rev. Bras. Ens. Fis. 43, e20210188 (2021).

[6] X. Wang e Z. Xia, em: International Conference on Modem Education and Information Management (Dalian, 2020).

[7] J.C. Santos e A.G. Dickman, Rev. Bras. Ens. Fis. 41, e20180161 (2019).

[8] C.N. Tulha, M.A.G. Carvalho e V.R. Coluci, Informática na Educação: Teoria \& Prática 22, 195 (2019).

[9] T.R. Barros e W.S. Dias, Rev. Bras. Ens. Fis. 41, e20190049 (2019)

[10] M.C.R. Pessanha, S.G. Cozendey e M.O. Souza, Rev. Bras. Ens. Fis. 32, 4503 (2010).

[11] N.L. Dias, A.G. Pinheiro e G.C. Barroso, Rev. Bras. Ens. Fis. 24, 232 (2002).

[12] V. Santos, S.R. Santos e L.M. Fraga, Rev. Bras. Ens. Fis. 24, 185 (2002).
[13] I. Yamamoto e V.B. Barbeta, Rev. Bras. Ens. Fis. 23, 215 (2001).

[14] D.C. Vilela, J.S.E. Germano, M.A.A. Monteiro e S.J. Carvalho, Rev. Bras. Ens. Fis. 41, e20190041 (2019).

[15] AGÊNCIA BRASIL, Brasil tem 134 milhões de usuários de internet, aponta pesquisa. Brasília, 2020. Disponível em: https://agenciabrasil.ebc.com.br/geral/noticia/202 0-05/brasil-tem-134-milhoes-de-usuarios-de-internetaponta-pesquisa

[16] P. Vieira, V.O.M. Lara e D.F. Amaral, Rev. Bras. Ens. Fis. 36, 3505 (2014).

[17] G. Hoff e N.W. Lima, em: International Joint Conference RADIO (Gramado, 2014).

[18] H.G. Gutierre, M.S. Ribeiro, L.A.A. Pereira, G.K. Cruz, R.F. Turchiello e S.L. Gómez, Rev. Bras. Ens. Fis. 39, e3501 (2017).

[19] J.A. Macêdo, L.S. Pedroso e G.A. Costa, Rev. Bras. Ens. Fis. 40, e5403 (2018)

[20] C.B. Pereira, S.L.M. Berleze, W.A. Soares e J.P.M. Serbena, Rev. Bras. Ens. Fis. 43, e20200502 (2021).

[21] T.D. Admiral, Rev. Bras. Ens. Fis. 42, e20200139 (2020).

[22] E. Paiva, Rev. Bras. Ens. Fis. 36, 3311 (2014).

[23] https://rexlab.ufsc.br/about/.

[24] M.A. Cavalcante, C.R.C. Tavolaro e E. Molisani, Rev. Bras. Ens. Fis. 33, 4503 (2011).

[25] M.P. Silva, V.B. Frota e M. Cavalcante, Brazilian Journal of Development 6, 37233 (2020).

[26] https://sites.google.com/view/fisetec/material/experim entação-remota/tecnologias.

[27] https://roboticsbackend.com/arduino-standard-firma ta-tutorial/

[28] https://github.com/IMSFisica/LDRvsLuz

[29] E.M.F. Santos, Arduino: uma ferramenta para aquisição de dados, controle e automação de experimentos de óptica em laboratório didático de física no ensino médio. Dissertação de Mestrado, Universidade Federal do Rio Grande do Sul, Porto Alegre (2014).

[30] https://docs.blender.org/manual/pt/dev/

[31] https://docs.unity3d.com/Manual/index.html

[32] M.D. Kickmeier-Rust, D. Schwarz, D. Albert, D. Verpoorten, J.L. Castaigne e M. Bopp, em: 2nd Symposium of the Workgroup Human-Computer Interaction and Usability Engineering (HCIधUE) (Vienna, 2006).

[33] C.S. Loh, International Journal of Gaming and Computer-Mediated Simulations 1, 4 (2009).

[34] https://play.google.com/store/apps/details?id=com.Iz acPhysics.LDR

[35] https://sites.google.com/view/fisetec/material/aplicati vos/tecnologias-app

[36] https://sites.google.com/view/fisetec/material/experim entação-remota/experimentos 